\title{
A Husband and a Wife with Simultaneous Presentation of Glioblastoma Multiforme: A Case Report
}

\author{
Giandomenico Roviello $^{a} \quad$ Roberto Petrioli $^{\mathrm{a}} \quad$ Alfonso Cerase ${ }^{\mathrm{b}}$ \\ Stefania Marsili ${ }^{a} \quad$ Clelia Miracco $^{c}$ Giovanni Rubino $^{d}$ Paolo Tini ${ }^{d}$ \\ ${ }^{a}$ Medical Oncology Unit, ${ }^{b}$ Unit NINT Neuroimaging and Neurointervention, Department of \\ Neurological and Sensorineural Sciences, 'Department of Human Pathology and \\ Oncology Section of Pathological Anatomy, and 'Section of Radiological Sciences, \\ Department of Human Pathology and Oncology, Azienda Opedaliera Universitaria Senese, \\ Policlinico 'Santa Maria alle Scotte', Siena, Italy
}

\section{Key Words}

Familial risk · Glioblastoma multiforme $\cdot$ Synchronous occurrence

\begin{abstract}
Glioblastoma multiforme (GBM) is the most lethal subtype of glioma, classified as a WHO grade 4 infiltrative glioma. The etiology of GBM remains unknown and risk factors can be identified only in a small minority. We report the synchronous occurrence of GBM in an otherwise unrelated married couple, i.e. a husband and his wife, who developed GBM within an interval of 1 month. No specific causative environmental factors were identified for both patients, and the genetic screens were negative for hereditary syndromes. Family history was negative for tumors, and no other incidence of cancer in either siblings, parents or other children was reported. An analysis of the couple's exposure to nonionizing electromagnetic fields and ionizing radiations revealed values within the normal ranges usually found in homes. Overall, conjugal tumors are rarely reported. However, the case reported herein raises important questions about possible etiologic factors.

(c) 2013 S. Karger AG, Basel
\end{abstract}


Roviello et al:: A Husband and a Wife with Simultaneous Presentation of Glioblastoma Multiforme: A Case Report

\section{Introduction}

Malignant gliomas (high-grade gliomas) are the most common primary brain tumors in adults, accounting for approximately $60 \%$ of them. Among these, glioblastoma multiforme (GBM) is the most lethal subtype of glioma, classified as a WHO grade 4 infiltrative glioma and associated with a median survival time of approximately 18 months as well as a poor response to chemotherapeutic drugs [1-3]. The etiology of GBM remains unknown and risk factors can be identified only in a small minority. Exposure to irradiation, especially in childhood, is the only risk factor that has been identified. Notably, among pediatric long-term survivors exposed to prophylactic cranial irradiation for acute leukemia, the incidence of primary brain tumors is $2.3 \%$, approximately a fourfold increase over the expected rate [4]. There are many hereditary syndromes affecting the CNS, and in these patients, GBM can occur in more than one related family member. However, the occurrence of GBM in unrelated spouses is unusual. The purpose of this case report is to describe an unrelated married couple who developed GBM within an interval of 1 month.

\section{Case Presentations}

\section{Patient 1}

The husband was a 76-year-old man who presented with headache, vague balance difficulties, left hemisensory and hemiparetic deficits in February 2012. He was admitted to another institution before he came to our institution, from which he was discharged with a clinical and neuroimaging diagnosis of hemorrhagic stroke (fig. 1a-c). Over the next 2 months, the patient did not complain about any discomfort except for mild-to-moderate left hemidysesthesia. In April 2012, a brain MRI showed resorption of the bleeding and clear evidence of a large, right parietal, extra-axial mass (fig. 1d, e). Platelet counts, prothrombin time and activated partial thromboplastin time were within normal ranges. The patient had no history of diabetes, hypertension or coagulation disorders and no history of trauma. He was a retired goldsmith living in a small town. His family history was negative for tumors and his two children were healthy. He pointed to his use of cell phones, which had started only a few years earlier, however, and occurred with moderate frequency. The patient underwent a subtotal resection of the intracranial mass through a right temporoparietal approach. Pathologic evaluation showed a GBM tumor cell proliferation index - as evaluated by anti-ki-67 MIB1 antibody (rabbit monoclonal antibody, diluted 1:100; NeoMarkers, Inc., Fremont, Calif., USA) - of about 55-65\%, an EGFR of about $65 \%$ and p53 was negative. The final pathologic diagnosis was grade IV GBM according to the WHO 2007 grading system. In May 2012, a brain MRI confirmed the lesion mass with a further bleeding. Considering the patient's clinical conditions and neuroimaging findings, we opted for supportive care and therapy with corticosteroids. No irradiation was performed. The patient continued supportive care waiting for the clinical conditions to allow treatment, but unfortunately, he died of his primary brain tumor in July 2012.

\section{Patient 2}

The wife was a 72-year-old woman who presented in March 2012 with ingravescent, vague balance difficulties. A brain MRI (fig. 2) showed a large, inhomogeneous, irregularly gadolinium-enhancing, right, paratrigonal, intra-axial mass. Additional findings included a macroadenoma and a small left frontal meningioma. The patient grew up in the same small town as her spouse, had always worked as a nurse and was retired at the time of diagnosis. 
Roviello et al.: A Husband and a Wife with Simultaneous Presentation of Glioblastoma Multiforme: A Case Report

She had been married for 46 years, with no evidence of possible consanguinity with her husband. Her family history was negative for tumors. She reported that use of cell phones had occurred only within the last 10 years, with a very moderate frequency and only upon need. The patient underwent subtotal resection of the intracranial mass. Pathologic evaluation showed a GBM tumor cell proliferation index - as evaluated by anti-ki-67 MIB1 antibody (rabbit monoclonal antibody, diluted 1:100; NeoMarkers, Inc., Fremont, Calif., USA) - of about 45-55\%; EGFR and p53 were negative. A diagnosis of grade IV GBM according to the WHO 2007 grading system was rendered. A follow-up brain MRI 1 month after surgery showed a volumetric increase of the right temporoparietal intra-axial mass with a mass effect on the ventricular system and midbrain, causing subfalcine herniation and a midline shift. Therefore, a second palliative surgery was performed. In May 2012, the patient started treatment with temozolomide (TMZ) in combination with adjuvant radiation therapy and corticosteroids. After she had finished radiotherapy, she was started on a 6month TMZ treatment. However, after only two cycles of TMZ, our patient went into a coma and died in July 2012.

\section{Discussion}

GBM is the most common and aggressive primary malignant brain tumor in humans and appears to occur most frequently in males and whites. Despite continuous advances in diagnosis, classification and therapy, prognosis is still poor. The median overall survival of $<18$ months is due to the resistance to radiotherapy and chemotherapy following surgery. Unlike other malignancies, GMB does not share common associations as others tumors do. Notably, the etiology and main risk factors leading to GMB are still mostly unknown.

Although most GBMs appear to be sporadic without any genetic predisposition, there are many hereditary syndromes affecting the CNS, including neurofibromatosis type 1 and 2 , Li-Fraumeni syndrome, von Hippel-Lindau syndrome, and Turcot's syndrome that seem to predispose to the development of other CNS tumors (astrocytomas, meningiomas and medulloblastoma) rather than GBM [5]. In both patients described herein, a genetic screening was performed and resulted negative for hereditary syndromes. However, as patient 2 had an associated pituitary macroadenoma and a meningioma, the occurrence of three intracranial tumors raised the question of their genetic determinance.

Other agents and specifically virus infections are also assumed to be involved in the development of brain tumors. Indeed, viral infections such as SV40 [6] or cytomegalovirus have already been suggested as a possible origin of malignant glioma. Furthermore, Lehrer [7] showed an increased incidence of brain tumors following malaria outbreaks in the United States and mentioned the anopheles mosquito as another possible source for brain tumors. In the patients reported herein, we were unable to clearly identify any virus as a risk factor, since the most important viral antigens were nonsignificant during the illness.

Exposure to toxins was also taken into consideration as a potential etiological factor in specific professions because a higher incidence of tumors has been registered in farmers and petrochemical workers, who are exposed to a variety of chemical products [8]. However, both patients had no history of occupational exposure. The husband's exposure in the processing of gold was uncertain and appeared irrelevant. A 24-urine screening for a metal test was performed in both spouses; however, there were no significant results.

The role of nonionizing radiation emitted by cell phones seems to be more controversial, since the link between the use of cell phones and brain tumors has been widely investigated. However, the results have no definitive conclusion. On May 31, 2011, the WHO announced 


\begin{tabular}{l|l}
\hline DOI: $10.1159 / 000356098$ & $\begin{array}{l}\text { C } 2013 \text { S. Karger AG, Basel } \\
\text { www.karger.com/cro }\end{array}$ \\
\hline
\end{tabular}

Roviello et al:: A Husband and a Wife with Simultaneous Presentation of Glioblastoma Multiforme: A Case Report

their classification of radiofrequency electromagnetic fields emitted from cell phones as 'possibly carcinogenic' [9]. However, a Danish study on the use of cell phones [10] performed with a large cohort of cancer patients from a national registry concluded that there was little evidence for a causal association and therefore no increased risk of CNS tumors. Another recent study by Little et al. [11] suggested that there is little evidence linking cell phone use to a higher risk for glioma. On the other hand, the researchers noted that the higher risks previously seen were not consistent with the incidence trends they found in the American population. Thus, the issue of the possible role of nonionizing radiation emitted by cell phones is far from been solved.

An analysis of exposure to nonionizing electromagnetic fields (transmitters, cell phones, radio alarm clocks, electrical appliances, electrical circuits) and ionizing radiations (natural radiations and possible contaminations) was performed in the couple's house and surrounding environment (furniture, tools, fields and orchards), but the results were within normal ranges.

Upon further investigation of the couple's habits, it was found that they both regularly used trichloroethylene solvents to clean the house floors. However, the role of this component appeared scarcely relevant, as it is currently not known to cause tumors of the brain [12].

As reported in the literature, the risk of simultaneous tumor occurrence is in the order of 1 in 185 million. The synchronous occurrence of GBM in a husband and a wife within an interval of 2 years has already been described in 1986 by Grifin et al. [13]. The year before, in 1985, Tupchong et al. [14] described the development of the same brain tumor in a husband and a wife within an interval of 18 months. The case reported herein is the first to describe the event occurring within an even shorter period of only 1 month. However, in all reported cases, the investigators were unable to identify the specific etiology, including the current report. Nonetheless, because the simultaneous occurrence of such tumors is so rare, the hypothesis of random occurrence of these tumors should be rejected.

In conclusion, we presented a case of synchronous occurrence of GBM in a husband and a wife that developed within an interval of 1 month. No specific cause was identified as environmental exposure was negative for risk factors such as electromagnetic fields and ionizing radiation, and there were no incidences of cancer in both patient families. To date, there are few data supporting the hypothesis of a common environmental exposure that predisposes couples to develop a brain tumor, but at the same time, it is very difficult to think of such simultaneously occurring tumors as a rare coincidence. Thus, further reports on similar cases are needed before an evident etiologic factor can be determined.

\section{References}

1 DeAngelis LM: Brain tumors. N Engl J Med 2001;344:114-123.

$\checkmark 2$ Stewart LA: Chemotherapy in adult high-grade glioma: a systematic review and meta-analysis of individual patient data from 12 randomised trials. Lancet 2002;359:1011-1018.

3 Behin A, Hoang-Xuan K, Carpentier AF, Delattre JY: Primary brain tumours in adults. Lancet 2003;361:323331.

4 Neglia JP, Meadow AT, Robison LL, et al: Second neoplasms after acute lymphoblastic leukemia in childhood. N Engl J Med 1991;325:1330-1336.

5 Ohgaki H, Kleihues P: Population-based studies on incidence, survival rates, and genetic alterations in astrocytic and oligodendroglial gliomas. J Neuropathol Exp Neurol 2005;64:479-489.

6 Miller G: Brain cancer. A viral link to glioblastoma? Science 2009;323:30-31.

7 Lehrer S: Anopheles mosquito transmission of brain tumor. Med Hypotheses 2010;74:167-168.

8 Ohgaki H, Kleihues P: Epidemiology and etiology of gliomas. Acta Neuropathol 2005;109:93-108.

-9 Monograph Working Group: Carcinogenicity of radiofrequency electromagnetic fields. Lancet Oncol 2011;12:624-625. 


\section{Case Reports in Oncology}

\begin{tabular}{l|l}
\hline Case Rep Oncol 2013;6:538-543 \\
\hline DOI: 10.1159/000356098 & $\begin{array}{l}\text { @ 2013 S. Karger AG, Basel } \\
\text { www.karger.com/cro }\end{array}$ \\
\hline
\end{tabular}

Roviello et al:: A Husband and a Wife with Simultaneous Presentation of Glioblastoma Multiforme: A Case Report

10 Frei P, Poulsen AH, Johansen C, Olsen JH, Steding-Jessen M, Schüz J: Use of mobile phones and risk of brain tumours: update of Danish cohort study. BMJ 2011;343:d6387.

11 Little MP, Rajaraman P, Curtis RE, Devesa SS, Inskip PD, Check DP, Linet MS: Mobile phone use and glioma risk: comparison of epidemiological study results with incidence trends in the United States. BMJ 2012;344:e1147.

12 Scott CS, Jinot J: Trichloroethylene and cancer: systematic and quantitative review of epidemiologic evidence for identifying hazards. Int J Environ Res Public Health 2011;8:4238-4272.

-13 Griffin TW, Smith TW, Levy BS, Recht LD: Synchronous occurrence of glioblastoma multiforme in a husband and wife. J Neurooncol 1986;4:75-78.

14 Tupchong L, Levison DA, Jones AE: Concomitant conjugal gliomas with similar histologic features. Cancer 1985;55:864-869.

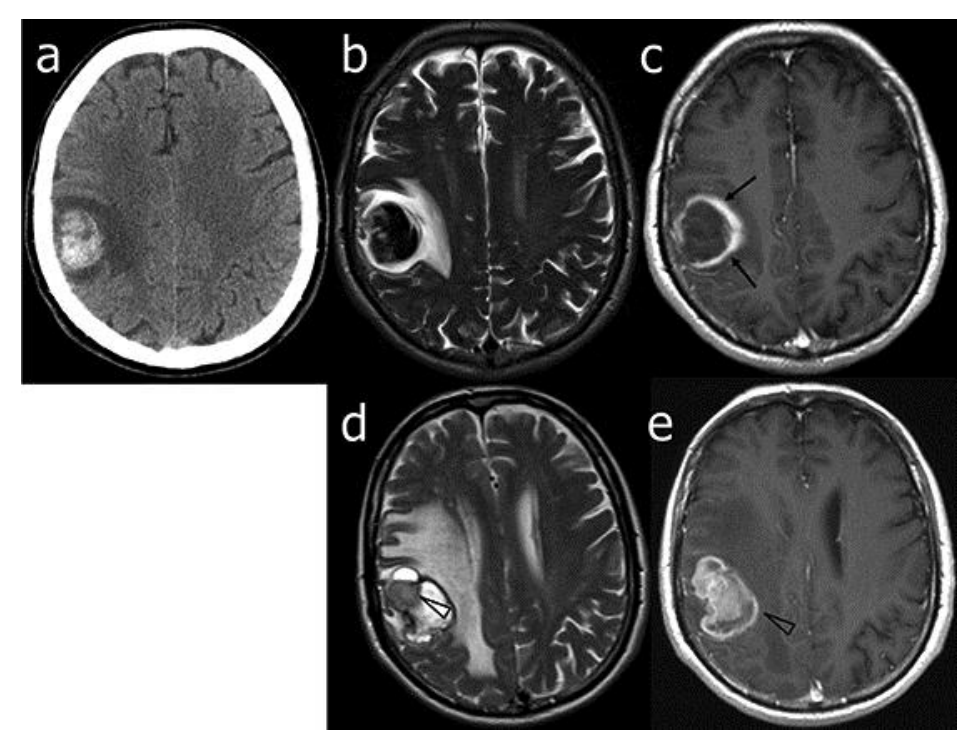

Fig. 1. Patient 1. Neuroimaging at diagnosis and follow-up. On admission, CT (a), T2-weighted (b) and gadolinium-enhanced T1-weighted (c) axial MR images showed an early, subacute, frontal hematoma with a different wall thickness (arrows) of uncertain nature as well as a vasogenic edema. Two months later, T2weighted (d) and gadolinium-enhanced T1-weighted (e) MR axial images showed only a slight reduction of the hematoma, an increase of the vasogenic edema and a clearcut evidence of gadolinium-enhanced pathologic solid tissue (arrowheads) consistent with GBM. 


\section{Case Reports in Oncology}

\begin{tabular}{l|l}
\hline Case Rep Oncol 2013;6:538-543 \\
\hline DOI: $10.1159 / 000356098$ & $\begin{array}{l}\text { C 2013 S. Karger AG, Basel } \\
\text { www.karger.com/cro }\end{array}$ \\
\hline
\end{tabular}

Roviello et al.: A Husband and a Wife with Simultaneous Presentation of Glioblastoma Multiforme: A Case Report

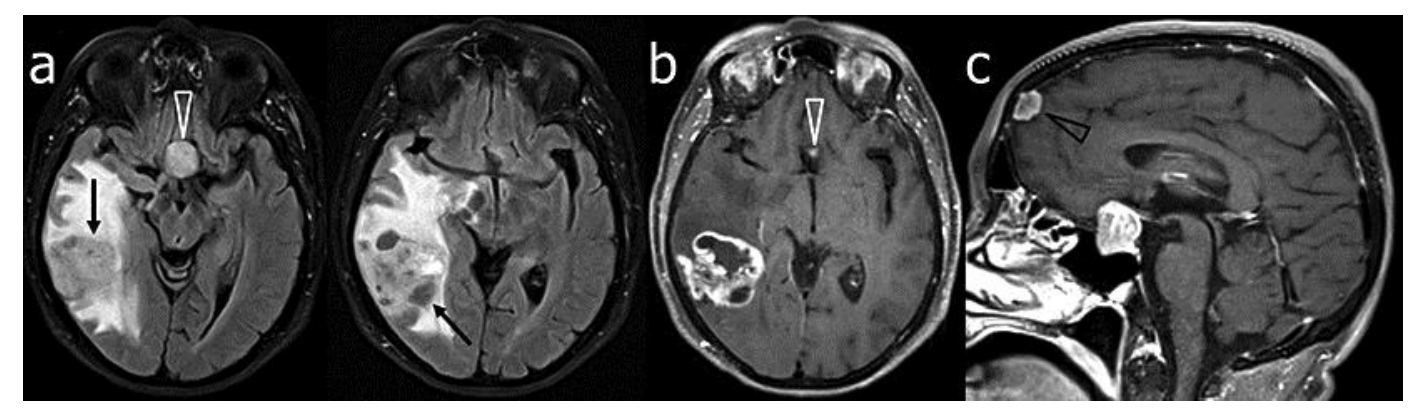

Fig. 2. Patient 2. MR images of the brain on admission. Serial consecutive fat-suppressed fluid-attenuated inversion recovery (a) and gadolinium-enhanced T1-weighted (b) axial images showed an inhomogeneous, irregularly contrast-enhancing mass lesion (arrows) in the right paratrigonal region associated with a marked vasogenic edema, resulting in a mass effect. Also note the sellar and suprasellar macroadenoma (white arrowheads), which is shown better on the gadolinium-enhanced T1-weighted sagittal image (c), and a left frontal meningioma (black arrowhead). 